\title{
ON THE SZLENK INDEX AND THE WEAK*-DENTABILITY INDEX
}

by Gilles LANCIEN (*)

EQUIPE D'ANALYSE

Université Paris VI

Boîte 186

4, Place Jussieu

75252 - PARIS CEDEX 05

France
Department of Mathematics

University of Missouri-Columbia

Columbia, Missouri 65211

U.S.A.

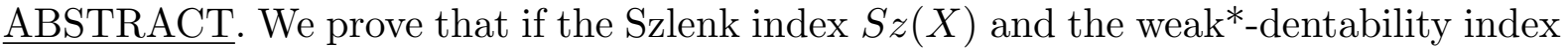
$\delta^{*}(X)$ of a Banach space $X$ are countable, then they are determined by the closed separable linear subspaces of $X$. From this we deduce the existence of an absolute function $\psi$ from $\omega_{1}$ to $\omega_{1}$ (first uncountable ordinal) such that $\delta^{*}(X)$ is bounded above by $\psi(S z(X))$, and that the condition $S z(X)<\omega_{1}$ yields the existence of an equivalent norm on $X$ whose dual norm is locally uniformly convex. As an other application, we compute $S z(C(K))$, where $K$ is a scattered compact space with $K^{\left(\omega_{1}\right)}=\emptyset$. Finally we solve the three space problem for the condition $S z(X)<\omega_{1}$.

(*) This research has been supported by a grant "Lavoisier" from the french "Ministère des Affaires Etrangères". 


\section{INTRODUCTION.}

Let $X$ be a Banach space. We will first define the two ordinal indices $\delta^{*}(X)$ and $S z(X)$. Weak*-dentability index, $\delta^{*}(X)$ :

Let $F$ be a closed bounded subset of $X^{*}$. For $\varepsilon>0, F_{\varepsilon}^{\prime}=\left\{x^{*} \in F\right.$ such that any weak ${ }^{*}$-slice of $F$ containing $x^{*}$ is of diameter $>\varepsilon$ \}.

For $\alpha$ ordinal we construct $F_{\varepsilon}^{\alpha}$ inductively :

$$
\begin{aligned}
& F_{\varepsilon}^{0}=F \\
& F_{\varepsilon}^{\alpha+1}=\left(F_{\varepsilon}^{\alpha}\right)_{\varepsilon}^{\prime} \\
& F_{\varepsilon}^{\alpha}=\bigcap_{\beta<\alpha} F_{\varepsilon}^{\beta}, \text { if } \alpha \text { is a limit ordinal. }
\end{aligned}
$$

Then

$$
\Delta_{\varepsilon}(F)= \begin{cases}\inf \left\{\alpha: F_{\varepsilon}^{\alpha}=\emptyset\right\} & \text { if it exists } \\ \infty & \text { otherwise }\end{cases}
$$

And $\Delta(F)=\sup _{\varepsilon>0} \Delta_{\varepsilon}(F)$.

Finally, we denote $\delta^{*}(X, \varepsilon)=\Delta_{\varepsilon}\left(B_{X^{*}}\right)$ and $\delta^{*}(X)=\Delta\left(B_{X^{*}}\right)$, where $B_{X^{*}}$ is the unit ball of $X^{*}$.

$\underline{\text { Szlenk index, } S z(X)}$ :

Let $F$ be a closed bounded subset of $X^{*}$. For $\varepsilon>0, F_{\varepsilon}^{\left[{ }^{\prime}\right]}=\left\{x^{*} \in F\right.$ such that for any weak*-neighborhood $V$ of $x^{*}$, $\left.\operatorname{diam}(V \cap F)>\varepsilon\right\}$.

We denote :

$$
\begin{gathered}
F_{\varepsilon}^{[0]}=F \\
\left.F_{\varepsilon}^{[\alpha+1]}=\left(F_{\varepsilon}^{[\alpha]}\right)_{\varepsilon}^{\left.{ }^{\prime}\right]}\right] \\
F_{\varepsilon}^{[\alpha]}=\bigcap_{\beta<\alpha} F_{\varepsilon}^{[\beta]}, \text { if } \alpha \text { is a limit ordinal. } \\
S_{\varepsilon}(F)= \begin{cases}\inf \left\{\alpha: F_{\varepsilon}^{[\alpha]}=\emptyset\right\} & \text { if it exists } \\
\infty & \text { otherwise }\end{cases} \\
S(F)=\sup _{\varepsilon>0} S_{\varepsilon}(F) \\
S z(X, \varepsilon)=S_{\varepsilon}\left(B_{X^{*}}\right) \text { and } S z(X)=S\left(B_{X^{*}}\right) .
\end{gathered}
$$

Clearly $S z(X) \leq \delta^{*}(X)$.

Our main objective is to prove that, for a Banach space $X, S z(X)<\omega_{1}$ if and only if $\delta^{*}(X)<\omega_{1}$, where $\omega_{1}$ is the first uncountable ordinal. Then, answering a question suggested by R. Haydon, we will be able to deduce that if $S z(X)<\omega_{1}$, then $X$ admits an equivalent norm whose dual norm is locally uniformly convex. An important step in the proof of this 
result is that, if $S z(X)$ is countable then $S z(X)=\sup \{S z(Y), Y$ closed separable subspace of $X\}$ and if $\delta^{*}(X)$ is countable then $\delta^{*}(X)=\sup \left\{\delta^{*}(Y), Y\right.$ closed separable subspace of $X$ \}. In section 5 we use this fact to compute $S z(C(K))$, for $K$ scattered compact space such that its $\omega_{1}^{t h}$ derived set $K^{\left(\omega_{1}\right)}$ is empty. In the last section of this paper we give a quantitative answer to the three space problem for the condition $S z(X)<\omega_{1}$.

\section{SEPARABLE CASE.}

It is well known that if $X$ is a separable Banach space, then the following are equivalent:

i) $S z(X)<\omega_{1}$

ii) $\delta^{*}(X)<\omega_{1}$

iii) $X^{*}$ is separable.

In this section we will explain how to obtain the following improvement.

PROPOSITION 2.1. There exists a function $\psi: \omega_{1} \rightarrow \omega_{1}$ so that, for any separable Banach space $X$ and for any $\alpha<\omega_{1}, S z(X) \leq \alpha$ implies $\delta^{*}(X) \leq \psi(\alpha)$.

This is the consequence of ideas developed by B. Bossard in a slightly different and also more general setting $[\mathrm{B}]$.

Before proceeding to the proof of this proposition, we will introduce a few notations :

Let $K=\left(B_{l^{\infty}}, \sigma\left(l^{\infty}, l^{1}\right)\right) . K$ is a compact metrizable space. We denote by $\mathcal{F}(K)$ the collection of all closed subsets of $K$ and we equip $\mathcal{F}(K)$ with the Hausdorff topology $\mathcal{T}$ generated by the sets of the form $\{F \in \mathcal{F}(K): F \cap V \neq \emptyset\}$ and $\{F \in \mathcal{F}(K): F \subset V\}$, where $V$ is an open subset of $K .(\mathcal{F}(K), \mathcal{T})$ is a compact metrizable space.

PROPOSITION 2.2. There exists a function $\psi: \omega_{1} \rightarrow \omega_{1}$ so that, for any closed subset $F$ of $K$ and for any $\alpha<\omega_{1}, S(F) \leq \alpha$ implies $\Delta(F) \leq \psi(\alpha)$.

Proof. We will need the following result of B. Bossard [B] :

$$
\begin{aligned}
& \text { for } \varepsilon>0 \quad d_{\varepsilon}: \mathcal{F}(K) \rightarrow \mathcal{F}(K) \quad \text { and } \quad D_{\varepsilon}: \mathcal{F}(K) \rightarrow \mathcal{F}(K) \\
& F \mapsto F_{\varepsilon}^{\prime} \quad F \mapsto F_{\varepsilon}^{\left[{ }^{\prime}\right]}
\end{aligned}
$$

are Borel derivations. 
Therefore, for any $\alpha<\omega_{1}, \mathcal{B}_{\alpha}=\{F \in \mathcal{F}(K): S(F) \leq \alpha\}=\bigcap_{n=1}^{\infty}\left\{F \in \mathcal{F}(K): S_{1 / n}(F) \leq \alpha\right\}$ is a Borel set in $(\mathcal{F}(K), \mathcal{T})$.

Moreover, for any $n \geq 1, \mathcal{B}_{\alpha} \subseteq\left\{F \in \mathcal{F}(K): \Delta_{1 / n}(F)<\omega_{1}\right\}$. Indeed, if $S(F)<\omega_{1}$, then $F$ is norm separable and therefore every weak*-closed subset of $F$ is weak*-dentable. So $\left(F_{1 / n}^{\alpha}\right)_{\alpha}$ is strictly decreasing and must stabilize at $\emptyset$ before $\omega_{1}$.

Now, by a result of C. Dellacherie [Del] about the applications of the Kunen-Martin theorem to the study of the analytic derivations, there exists $\psi_{n}(\alpha)<\omega_{1}$ such that:

$$
\mathcal{B}_{\alpha} \subseteq\left\{F \in \mathcal{F}(K): \Delta_{1 / n}(F) \leq \psi_{n}(\alpha)\right\}
$$

We can conclude the proof by taking $\psi(\alpha)=\sup _{n \geq 1} \psi_{n}(\alpha)$.

Proof of Proposition 2.1. Let $X$ be a separable Banach space and $\alpha<\omega_{1}$.

There is a closed linear subspace $Y$ of $\ell_{1}(\mathbb{N})$ such that $X$ is isomorphic to $\frac{\ell_{1}(\mathbb{N})}{Y}$.

So $S z(X)=S z\left(\frac{\ell_{1}(\mathbb{N})}{Y}\right)=S\left(B_{Y^{\perp}}\right)$

and $\delta^{*}(X)=\delta^{*}\left(\frac{\ell_{1}(\mathbb{N})}{Y}\right)=\Delta\left(B_{Y^{\perp}}\right)$.

Thus by proposition 2.2 , if $S z(X) \leq \alpha$, then $\delta^{*}(X) \leq \psi(\alpha)$.

3. WHEN COUNTABLE, $S z(X)$ AND $\delta^{*}(X)$ ARE SEPARABLY DETERMINED.

Our goal in this section is to prove the two following statements :

PROPOSITION 3.1. Let $X$ be a Banach space and let $\alpha<\omega_{1}$.

If $S z(X)>\alpha$, then there exists a separable closed subspace $Y$ of $X$ such that $S z(Y)>\alpha$.

PROPOSITION 3.2. Let $X$ be a Banach space and let $\alpha<\omega_{1}$.

If $\delta^{*}(X)>\alpha$, then there exists a separable closed subspace $Y$ of $X$ such that $\delta^{*}(Y)>\alpha$. 
Proof of Proposition 3.1: We will give our original proof in which we construct "by hand" the space $Y$. In order to do this we will use a family $\left(T_{\alpha}\right)_{\alpha<\omega_{1}}$ of trees on $\omega$ (first infinite ordinal) constructed inductively in the following way :

$$
\begin{aligned}
& T_{0}=\{\emptyset\} \\
& T_{\alpha+1}=\{\emptyset\} \cup \bigcup_{n=0}^{\infty} n \frown T_{\alpha}, \text { where } n \frown T_{\alpha}=\left\{n \frown s, s \in T_{\alpha}\right\} . \\
& T_{\alpha}=\{\emptyset\} \cup \bigcup_{n=0}^{\infty} n \frown T_{\alpha_{n}}, \text { if } \alpha \text { is a limit ordinal, }\left(\alpha_{n}\right)_{n=0}^{\infty} \text { being an enumeration of the }
\end{aligned}
$$
ordinals less than $\alpha$.

\section{$\underline{\text { Remarks : }}$}

1) The height of $T_{\alpha}$ is $h t\left(T_{\alpha}\right)=\alpha$.

2) For $s$ in $T_{\alpha}$ we denote $T_{\alpha}(s)=\left\{t \in \omega^{<\omega}: s \frown t \in T_{\alpha}\right\}$, where $\omega^{<\omega}$ is the set of all finite sequences of elements of $\omega$. If we call $h_{\alpha}(s)=h t\left(T_{\alpha}(s)\right)$, we have that $T_{\alpha}(s)=T_{h_{\alpha}(s)}$.

We will need the following :

LEMMA 3.3. For any $1 \leq \alpha<\omega_{1}$, there exists a bijection $\varphi_{\alpha}: \omega \rightarrow T_{\alpha}$ such that :

For any $s, s^{\prime}$ in $T_{\alpha}, s<s^{\prime}$ implies $\varphi_{\alpha}^{-1}(s)<\varphi_{\alpha}^{-1}\left(s^{\prime}\right)$.

Proof. Let $\left\{\mathcal{B}_{n}\right\}_{n=0}^{\infty}$ be an enumeration of the branches of $T_{\alpha}$. In order to define $\varphi_{\alpha}$ we enumerate successively $\mathcal{B}_{1}, \mathcal{B}_{2} \backslash \mathcal{B}_{1}, \ldots, \mathcal{B}_{n+1} \backslash \bigcup_{k=1}^{\infty} \mathcal{B}_{k}, \ldots$ (each enumeration of $\mathcal{B}_{n+1} \backslash \bigcup_{k=1}^{\infty} \mathcal{B}_{k}$ following the natural partial order on $T_{\alpha}$ ).

LEMMA 3.4. Let $1 \leq \alpha<\omega_{1}$ and $\varepsilon>0$ and let $X$ be a Banach space. If $x^{*} \in\left(B_{X^{*}}\right)_{\varepsilon}^{[\alpha]}$, then there exist a separable subspace $Y$ of $X$ and a family $\left(x_{s}^{*}\right)_{s \in T_{\alpha}} \subseteq B_{X^{*}}$ such that

i) $x_{\emptyset}^{*}=x^{*}$

ii) $\forall s \in\left(T_{\alpha}\right)^{\prime}, \forall n \in \omega:\left\|\left(x_{s \frown n}^{*}-x_{s}^{*}\right)_{\Gamma_{Y}}\right\|>\frac{\varepsilon}{2}$.

iii) $\forall s \in\left(T_{\alpha}\right)^{\prime}:\left(x_{s \frown n}^{*}-x_{s}^{*}\right)_{\Gamma_{Y}} \stackrel{\sigma\left(Y^{*}, Y\right)}{\longrightarrow} 0$.

(Note $:\left(T_{\alpha}\right)^{\prime}=\left\{s \in T_{\alpha}, \exists n \in \omega: s \frown n \in T_{\alpha}\right\}$ ). 
$\underline{\text { Proof. }}$ We will construct, by induction on $n,\left(x_{\varphi_{\alpha}(n)}^{*}\right)_{n=0}^{\infty}$ in $B_{X^{*}}$ and $\left(x_{n}\right)_{n=1}^{\infty}$ in $B_{X}$ so that :

a) $x_{\varphi_{\alpha}(0)}^{*}=x_{\emptyset}^{*}=x^{*}$.

b) $\forall n \in \omega, x_{\varphi_{\alpha}(n)}^{*} \in\left(B_{X^{*}}\right)_{\varepsilon}^{\left[h_{\alpha}\left(\varphi_{\alpha}(n)\right)\right]}$

c) $\forall n \geq 1,\left(x_{\varphi_{\alpha}(n)}^{*}-x_{s_{n}}^{*}\right)\left(x_{n}\right)>\frac{\varepsilon}{2}$, where $\varphi_{\alpha}(n)=s_{n} k_{n}$ with $k_{n} \in \omega$.

d) $\forall n \geq 2, \forall 1 \leq k \leq n-1,\left|\left(x_{\varphi_{\alpha}(n)}^{*}-x_{s_{n}}^{*}\right)\left(x_{k}\right)\right| \leq \frac{1}{2^{n}}$.

Assume $x_{\varphi_{\alpha}(k)}^{*}$ for $0 \leq k \leq n-1$ and $x_{k}$ for $1 \leq k \leq n-1$ have been constructed and satisty a)...d). By Lemma 3.3, there exists $i_{n}<n$ such that $\varphi_{\alpha}(n)=$ $\varphi_{\alpha}\left(i_{n}\right) \frown k_{n}$, with $k_{n} \in \omega$. By induction hypothesis $x_{\varphi_{\alpha}\left(i_{n}\right)}^{*} \in\left(B_{X^{*}}\right)_{\varepsilon}^{\left[h_{\alpha}\left(\varphi_{\alpha}\left(i_{n}\right)\right)\right]}$. Since $h_{\alpha}\left(\varphi_{\alpha}\left(i_{n}\right)\right) \geq h_{\alpha}\left(\varphi_{\alpha}(n)\right)+1$, we have that $x_{\varphi_{\alpha}\left(i_{n}\right)}^{*} \in\left(B_{X^{*}}\right)_{\varepsilon}^{\left[h_{\alpha}\left(\varphi_{\alpha}(n)\right)+1\right]}$. So for any weak*neighborhood $V$ of $x_{\varphi_{\alpha}\left(i_{n}\right)}^{*}$, diam $\left(V \cap\left(B_{X^{*}}\right)_{\varepsilon}^{\left[h_{\alpha}\left(\varphi_{\alpha}(n)\right)\right]}\right)>\varepsilon$. In particular there exists $x_{\varphi_{\alpha}(n)}^{*} \in\left(B_{X^{*}}\right)_{\varepsilon}^{\left[h_{\alpha}\left(\varphi_{\alpha}(n)\right)\right]}$ such that : $\left\|x_{\varphi_{\alpha}(n)}^{*}-x_{\varphi_{\alpha}\left(i_{n}\right)}^{*}\right\|>\frac{\varepsilon}{2}$ and $\left|\left(x_{\varphi_{\alpha}(n)}^{*}-x_{\varphi_{\alpha}\left(i_{n}\right)}^{*}\right)\left(x_{k}\right)\right| \leq \frac{1}{2^{n}}, \forall 1 \leq k \leq n-1$.

We conclude the induction by choosing $x_{n}$ in $B_{X}$ such that $\left(x_{\varphi_{\alpha}(n)}^{*}-x_{\varphi_{\alpha}\left(i_{n}\right)}^{*}\right)\left(x_{n}\right)>\frac{\varepsilon}{2}$.

Let $Y$ be the closed linear span of $\left\{x_{n}\right\}_{n=1}^{\infty}$. $Y$ and the family $\left(x_{s}^{*}\right)_{s \in T_{\alpha}}$ constructed by induction satisfy the properties claimed in Lemma 3.4 .

It is now easy to show that $x_{\Gamma_{Y}}^{*} \in\left(B_{Y^{*}}\right)_{\varepsilon / 2}^{[\alpha]}$. This completes the proof of Proposition 3.1.

Proof of Proposition 3.2: It is possible, by using convex combinations, to adapt the proof of Proposition 3.1. But we will use instead a simpler and more global technique that has been indicated to us.

We will show by transfinite induction that for any countable ordinal $\alpha$, there is a separable subspace $Z_{\alpha}$ of $X$ such that for any $\gamma \leq \alpha: x^{*} \in\left(B_{X^{*}}\right)_{\varepsilon}^{\gamma}$ implies $x_{I Z_{\alpha}}^{*} \in\left(B_{Z_{\alpha}^{*}}\right)_{\varepsilon}^{\gamma}$. First we pick $x$ in $X \notin\{0\}$ and call $Z_{0}=\mathbb{R} x$.

Assume that the previous statement is true for any $\beta<\alpha$.

If $\alpha$ is a limit ordinal, we choose $Z_{\alpha}$ to be the closed linear span of $\bigcup_{\beta<\alpha} Z_{\beta}$.

If $\alpha=\beta+1$ : let us call $V_{0}=Z_{\beta}$. Let $D_{0}$ be a countable dense subset of $V_{0}$ and $\mathcal{S}_{0}$ be the collection of half spaces $S=\left\{x^{*} \in X^{*}: x^{*}(z)>q\right\}$ with $z$ in $D_{0}$ and $q$ in $\mathbb{Q}$ If $S \cap\left(B_{X^{*}}\right)_{\varepsilon}^{\gamma+1} \neq \emptyset$ for some $\gamma \leq \beta$, then $\operatorname{diam}\left(S \cap\left(B_{X^{*}}\right)_{\varepsilon}^{\gamma}>\varepsilon\right.$ and therefore we can find $u^{*}, v^{*}$ in $S \cap\left(B_{X^{*}}\right)_{\varepsilon}^{\gamma}$ and $x=x(\gamma, S)$ in $B_{X}$ such that $\left(u^{*}-v^{*}\right)(x)>\varepsilon$. 
Let us denote by $V_{1}$ the closed linear span of $Z_{\beta} \cup \bigcup_{\gamma \leq \beta} \bigcup_{S \in \mathcal{S}_{0}} x(\gamma, S)$.

Then we consider $D_{1}$ a countable dense subset of $V_{1}$ and we construct $V_{2}$ similarly.

Finally $Z_{\alpha+1}$ is the closed linear span of $\bigcup_{n=0}^{\infty} V_{n}$.

We now need to prove by induction that for any $\gamma \leq \alpha: x^{*} \in\left(B_{X^{*}}\right)_{\varepsilon}^{\gamma}$ implies $x_{I Z_{\alpha}}^{*} \in\left(B_{Z_{\alpha}^{*}}\right)_{\varepsilon}^{\gamma}$. The case $\gamma=0$ and the limit case are trivial, so let us assume that this is true for $\gamma$.

Let $x^{*} \in\left(B_{X^{*}}\right)_{\varepsilon}^{\gamma+1}$ and let $S$ be a slice of $\left(B_{Z_{\alpha}^{*}}\right)_{\varepsilon}^{\gamma}$ containing $x^{*}$. We may assume that $S$ is defined by a $z$ in some $D_{n}$ and by a $q$ in Q Let $u^{*}$ and $v^{*}$ in $S \cap\left(B_{X^{*}}\right)_{\varepsilon}^{\gamma}$ such that $\left(u^{*}-v^{*}\right)(x(\gamma, S))>\varepsilon$.

By induction hypothesis $u_{\Gamma Z_{\alpha}}^{*}$ and $v_{I Z_{\alpha}}^{*}$ belong to $\left(B_{Z_{\alpha}^{*}}\right)_{\varepsilon}^{\gamma}$.

Thus $\operatorname{diam}\left(S \cap\left(B_{Z_{\alpha}^{*}}\right)_{\varepsilon}^{\gamma}\right)>\varepsilon$ and $x_{\Gamma Z_{\alpha}}^{*} \in\left(B_{Z_{\alpha}^{*}}\right)_{\varepsilon}^{\gamma+1}$.

Remark: This method gives similar results about ordinals with a different cardinality and the subspaces of $X$ with corresponding density character.

However a refinement of the technique used in the proof of Proposition 3.1. allows us to obtain the following extension :

PROPOSITION 3.5. Let $X$ be a Banach space with a separable dual and let $\alpha<\omega_{1}$. If $S z(X)>\alpha$, then there is a subspace $Z$ of $X$ such that $\frac{X}{Z}$ has a shrinking basis and $S z\left(\frac{X}{Z}\right)>\alpha$.

Proof : It will follow from a slight modification of W.B. Johnson and H.P. Rosenthal's proof of the existence of a quotient with a shrinking basis for any Banach space with separable dual $([\mathrm{J}-\mathrm{R}])$.

Since $X^{*}$ is separable, we may assume that the norm of $X$ is such that the weak* and the norm topologies coincide on the unit sphere of $X^{*}$.

Let $\varepsilon>0$ such that $0 \in\left(B_{X^{*}}\right)_{2 \varepsilon}^{[\alpha]},\left(\varepsilon_{n}\right)_{n \geq 1} \subseteq(0,1)$ such that $\sum_{n=1}^{\infty} \varepsilon_{n}<\infty$ and $\left(x_{n}\right)_{n \geq 1}$ be a dense subset of $B_{X}$. We will construct by induction $\left(x_{\varphi_{\alpha}(n)}^{*}\right)_{n=0}^{\infty} \subseteq B_{X^{*}}$ and $\left(F_{n}\right)_{n=1}^{\infty}$ an increasing sequence of finite subsets $B_{X}$ verifying :

a) $x_{\varphi_{\alpha}(0)}^{*}=0$.

b) $\forall n \geq 0, x_{\varphi_{\alpha}(n)}^{*} \in\left(B_{X^{*}}\right)_{2 \varepsilon}^{\left[h_{\alpha}\left(\varphi_{\alpha}(n)\right)\right]}$. 
c) $\forall n \geq 1,\left\|x_{\varphi_{\alpha}(n)}^{*}-x_{s_{n}}^{*}\right\|>\varepsilon,\left(\right.$ let us denote $\left.y_{n}^{*}=\frac{x_{\varphi_{\alpha}(n)}^{*}-x_{s_{n}}^{*}}{\left\|x_{\varphi_{\alpha}(n)}^{*}-x_{s_{n}}^{*}\right\|}\right)$.

d) For any $f$ in $\left(\left[y_{k}^{*}\right]_{k=1}^{n}\right)^{*}$ with $\|f\| \leq 1$, there is $x \in F_{n}$ such that :

$$
\forall y^{*} \in\left[y_{k}^{*}\right]_{k=1}^{n},\left|f\left(y^{*}\right)-y^{*}(x)\right|<\frac{\varepsilon_{n}}{3}\left\|y^{*}\right\| \text {. }
$$

e) $\forall x \in F_{n} \quad\left|y_{n+1}^{*}(x)\right| \leq \frac{\varepsilon_{n}}{3}$.

f) $\forall n \geq 1 \quad\left(x_{k}\right)_{k=1}^{n} \subseteq F_{n}$.

Suppose $\left(x_{\varphi_{\alpha}(k)}^{*}\right)_{k=0}^{n}$ and $F_{n-1}$ have been constructed. Take $F_{n}$ satisfying d) and f). As in the proof of Proposition 3.1, we now choose $x_{\varphi_{\alpha}(n+1)}^{*}$ in $\left(B_{X^{*}}\right)_{2 \varepsilon}^{\left[h_{\alpha}\left(\varphi_{\alpha}(n+1)\right)\right]}$ such that $\left\|x_{\varphi_{\alpha}(n)}^{*}-x_{s_{n}}^{*}\right\|>\varepsilon$ and $\left|y_{n+1}^{*}(x)\right| \leq \frac{\varepsilon_{n}}{3}$ for all $x$ in $F_{n}$.

Consequences of this construction : By d) and e), $\left(y_{n}^{*}\right)_{n=1}^{\infty}$ is a basic sequence in $X^{*}$ and, if we denote by $P_{n}$ the natural projections from $\left[y_{k}^{*}\right]_{k=1}^{\infty}$ onto $\left[y_{k}^{*}\right]_{k=1}^{n}$, we have that $\left\|P_{n}\right\| \rightarrow 1$. Let $\left(y_{k}\right)_{k=1}^{\infty} \subseteq\left(\left[y_{k}^{*}\right]_{k=1}^{\infty}\right)^{*}$ be the biorthogonal functionnals associated to the basis $\left(y_{k}^{*}\right)_{k=1}^{\infty}$. Following the paper of W.B. Johnson and H.P. Rosenthal ([J-R]) it is now possible to check that the operator :

$$
\begin{aligned}
T: X & \rightarrow\left(\left[y_{k}^{*}\right]_{k=1}^{\infty}\right)^{*} \\
x & \mapsto T x, \text { where } T x\left(y^{*}\right)=y^{*}(x)
\end{aligned}
$$

maps $X$ onto $Y$ the closed linear span of $\left(y_{k}\right)_{k=1}^{\infty}$. From this we can deduce, as in [J-R], that $\left(y_{k}^{*}\right)_{k=1}^{\infty}$ is a weak ${ }^{*}$-basic sequence. Finally, since the norm and the weak ${ }^{*}$ topologies coincide on the unit sphere of $X^{*}$ we can see, still following $[\mathrm{J}-\mathrm{R}]$, that $\left(y_{k}^{*}\right)_{k=1}^{\infty}$ is boundedly complete. Therefore, $\left(y_{k}\right)_{k=1}^{\infty}$ is shrinking.

Moreover our construction insures that $S z(Y)>\alpha$. Thus we can conclude the proof by taking $Z=\operatorname{Ker} T$.

\section{MAIN RESULTS.}

THEOREM 4.1. There exists a function $\psi: \omega_{1} \rightarrow \omega_{1}$ so that, for any Banach space $X$ and for any countable ordinal $\alpha: S z(X) \leq \alpha$ implies $\delta^{*}(X) \leq \psi(\alpha)$.

Proof. This is an immediate consequence of Proposition 3.2 and Proposition 2.1. The function $\psi$ is the same as the function given by Proposition 2.1. 


\section{Remarks.}

1) For a Banach space $X$, it is possible to define a dentability index $\delta(X)$ and a "weakSzlenk" index $S z_{\omega}(X)$ by peeling the unit ball of $X$ with slices of small diameter or with weakly open sets of small diameter. But the two conditions " $\delta(X)<\omega_{1}$ " and " $S z_{\omega}(X)<\omega_{1}$ " are not equivalent, even in the separable case. Indeed the predual $B$ of the James tree space has the Point of Continuity Property and is separable, so $S z_{\omega}(B)<\omega_{1}$; but $B$ does not have the Radon-Nikodym Property, so $\delta(X)=\infty$ (see R.C. James [J], J. Lindenstrauss and C. Stegall [L-S], C.A. Edgar and R.F. Wheeler [E-W]).

2) In general $\psi(\alpha)>\alpha$. For instance, if $X$ is finite dimensional, $S z(X)=1$, while $\delta^{*}(X)=\omega$. Moreover, the condition $\delta^{*}(X)=\omega$ is equivalent to $X$ super reflexive. But this is not true for the Szlenk index. For example it is easy to check that $S z\left(\left(\sum_{n=1}^{\infty} l_{1}^{n}\right)_{l_{2}}\right)=\omega$.

On the other hand, the descriptive set theory approach used in section 2 implies that

$$
\left\{\alpha<\omega_{1}:\{X \text { Banach space }: S z(X)<\alpha\}=\left\{X \text { Banach space }: \delta^{*}(X)<\alpha\right\}\right\}
$$

contains a closed cofinal subset of $\omega_{1}$.

THEOREM 4.2. Let $X$ be a Banach space. If $S z(X)<\omega_{1}$, then $X$ admits an equivalent norm whose dual norm is locally uniformly convex. In particular, there is an equivalent Fréchet-differentiable norm on $X$.

Proof. This result is proven in $[\mathrm{L}]$ under the "a priori" stronger hypothesis : $\delta^{*}(X)<$ $\omega_{1}$.

\section{5. $S z(\mathcal{C}(K))$ for $K$ SCATTERED COMPACT SPACE.}

For a topological space $K$, the derived space $K^{\prime}$ is defined to be $K \backslash\{x: x$ isolated point of $K\}$; for ordinals $\alpha$ we define $K^{(\alpha)}$ inductively by $K^{(0)}=K, K^{(\alpha+1)}=\left(K^{(\alpha)}\right)^{\prime}, K^{(\alpha)}=$ $\bigcap_{\beta<\alpha} K^{(\beta)}$ for $\alpha$ limit ordinal. Then the space $K$ is said to be scattered if $K^{(\alpha)}=\emptyset$ for some $\alpha$.

THEOREM 5.1. Let $K$ be a scattered compact space such that $K^{\left(\omega_{1}\right)}=\emptyset$. Let $\alpha<\omega_{1}$ be the ordinal such that $K^{\left(\omega^{\alpha}\right)} \neq \emptyset$ and $K^{\left(\omega^{\alpha+1}\right)}=\emptyset$. Then $S z(\mathcal{C}(K))=\omega^{\alpha+1}$. 
As a corollary we obtain the following result of $\mathrm{R}$. Deville [Dev] : if $K$ is a compact space such that $K^{\left(\omega_{1}\right)}=\emptyset$, then there is an equivalent norm on $\mathcal{C}(K)$ whose dual norm is locally uniformly convex.

We will need two classical lemmas.

LEMMA 5.2. Let $K$ be a compact space and $X$ be a separable subspace of $\mathcal{C}(K)$. Then there exists a compact space $L$ such that :

i) $\mathcal{C}(L)$ is separable.

ii) $X$ embeds isometrically into $\mathcal{C}(L)$.

iii) there is a map $s: K \rightarrow L$ which is continuous and onto.

Proof. Let $X$ be a separable subspace of $\mathcal{C}(K)$. We denote by $L_{0}$ the metrizable compact space $\left(B_{X^{*}}, \sigma\left(X^{*}, X\right)\right)$. For $x$ in $K$ we call $\delta_{x}$ the element of $(\mathcal{C}(K))^{*}$ defined by : for any $f$ in $\mathcal{C}(K), \delta_{x}(f)=f(x)$.

We have that the application $s: K \rightarrow L_{0}$

$$
x \mapsto \delta_{x} I_{X} \quad \text { is continuous. }
$$

Let $L=s(K)$. $X$ embeds isometrically and in a canonical way into $\mathcal{C}(L)$.

LEMMA 5.3. Let $K$ and $L$ be two compact spaces and let $s: K \rightarrow L$ be continous and onto. Then, for any ordinal $\alpha, L^{(\alpha)} \subseteq s\left(K^{(\alpha)}\right)$.

The proof of this lemma is an easy transfinite induction.

Proof of theorem 5.1. Let $K$ be a compact space such that $K^{\left(\omega^{\alpha+1}\right)}=\emptyset$, with $\alpha<\omega_{1}$. Let $X$ be a separable subspace of $\mathcal{C}(K)$. By Lemma 5.2, there is a compact space $L$ such that $\mathcal{C}(L)$ is separable, $X$ embeds isometrically in $\mathcal{C}(L)$ and there is a continuous map $s$ from $K$ onto $L$. Then by Lemma $5.3, L^{\left(\omega^{\alpha+1}\right)}=\emptyset$. Since $\mathcal{C}(L)$ is separable and $L$ is scattered we have that $L$ is countable. Now, it is known that for countable compact spaces, $L^{\left(\omega^{\alpha+1}\right)}=\emptyset$ implies $S z(\mathcal{C}(L)) \leq \omega^{\alpha+1}$ (see C. Samuel [Sa]). Therefore, for any separable subspace $X$ of $\mathcal{C}(K), S z(X) \leq \omega^{\alpha+1}$. Thus, by Proposition 3.1, $S z(\mathcal{C}(K)) \leq \omega^{\alpha+1}$.

Let us mention that the definition of the Szlenk index that we use is not the definition introduced by W. Szlenk $[\mathrm{Sz}]$ and used by C. Samuel [Sa]. But the two definitions coincide 
for $X$ separable Banach space not containing any isomorphic copy of $l_{1}(\mathbb{N})$ (see $[\mathrm{L}]$ ) and therefore for $\mathcal{C}(L)$ with $L$ countable compact space.

On the other hand, if $K^{\left(\omega^{\alpha}\right)} \neq \emptyset$, then $S z(\mathcal{C}(K))>\omega^{\alpha}$. More precisely we have that, for any ordinal $\alpha: x \in K^{(\alpha)} \Rightarrow \delta_{x} \in\left(B_{\mathcal{C}(K)^{*}}\right)_{1}^{[\alpha]}$, where $\delta_{x}$ is the point evaluation at $x$.

Therefore, under the assumptions of theorem 5.1 we have that $\omega^{\alpha}<S z(\mathcal{C}(K)) \leq \omega^{\alpha+1}$. The conclusion of this proof follows immediately from the next proposition.

PROPOSITION 5.4. Let $X$ be a Banach space such that $S z(X)<\omega_{1}$. Then there exists a countable ordinal $\alpha$ so that $S z(X)=\omega^{\alpha}$.

Proof. We will use the following fact : for any Banach space $X$ and any ordinal $\alpha$

$$
\frac{1}{2}\left(B_{X^{*}}\right)_{\varepsilon}^{[\alpha]}+\frac{1}{2} B_{X^{*}} \subseteq\left(B_{X^{*}}\right)_{\varepsilon / 2}^{[\alpha]}
$$

The proof of this is a straightforward transfinite induction.

Claim : $S z(X)>\omega^{\alpha} \Rightarrow S z(X) \geq \omega^{\alpha+1}$.

If $S z(X)>\omega^{\alpha}$ then we can find $\varepsilon>0$ and $x^{*} \in B_{X^{*}}$ such that $x^{*} \in\left(B_{X^{*}}\right)_{2 \varepsilon}^{\left[\omega^{\alpha}\right]}$. Then, by $(*), 0 \in\left(B_{X^{*}}\right)_{\varepsilon}^{\left[\omega^{\alpha}\right]}$.

Thus $\frac{1}{2} B_{X^{*}} \subseteq\left(B_{X^{*}}\right)_{\varepsilon / 2}^{\left[\omega^{\alpha}\right]}$. So $\left(\frac{1}{2} B_{X^{*}}\right)_{\varepsilon / 2}^{\left[\omega^{\alpha}\right]} \subseteq\left(B_{X^{*}}\right)_{\varepsilon / 2}^{\left[\omega^{\alpha} \cdot 2\right]}$. But $0 \in\left(B_{X^{*}}\right)_{\varepsilon}^{\left[\omega^{\alpha}\right]} \Rightarrow 0 \in$ $\left(\frac{1}{2} B_{X^{*}}\right)_{\varepsilon / 2}^{\left[\omega^{\alpha}\right]}$. Hence $0 \in\left(B_{X^{*}}\right)_{\varepsilon / 2}^{\left[\omega^{\alpha} \cdot 2\right]}$.

Proceeding inductively, we show that for any $n$ in $\omega, 0 \in\left(B_{X^{*}}\right)_{\varepsilon / 2^{n}}^{\left[\omega^{\alpha \cdot 2^{n}}\right]}$.

Therefore $S z(X) \geq \omega^{\alpha+1}$. This completes the proof of the Claim.

Now let $\alpha=\operatorname{Inf}\left\{\gamma: S z(X) \leq \omega^{\gamma}\right\}$. If $\alpha$ is a limit ordinal, $S z(X) \geq \sup _{\beta<\alpha} \omega^{\beta}=\omega^{\alpha}$. So $S z(X)=\omega^{\alpha}$. If $\alpha=\beta+1$, our claim implies that $S z(X)=\omega^{\alpha}$.

\section{$\underline{\text { Remarks. }}$}

1) A similar argument shows that if $\delta^{*}(X)<\omega_{1}$, then $\delta^{*}(X)$ is of the form $\omega^{\alpha}$.

2) The property described by Proposition 5.4 has been suggested by a paper of A. Sersouri [Se] about the Lavrientiev indices. 


\section{THREE-SPACE PROBLEM FOR THE CONDITION $S z(X)$ COUNTABLE.}

The general question we are now interested in is the following : let $X$ be a Banach space and $Y$ be a closed subspace of $X$. Assume that $S z(Y)<\omega_{1}$ and $S z(X / Y)<\omega_{1}$. Can we conclude that $S z(X)<\omega_{1}$ ?

In this section we answer positively this question by proving the following result :

THEOREM 6.1. Let $X$ be a Banach space and $Y$ be a closed subspace of $X$ such that $S z(Y)<\omega_{1}$ and $S z(X / Y)<\omega_{1}$. Then $S z(X) \leq S z(X / Y) . S z(Y)$

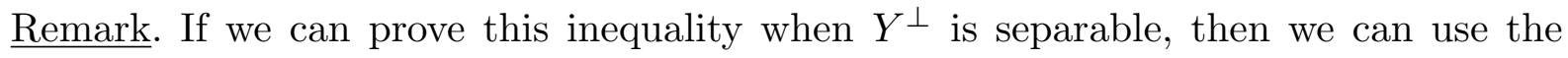
results of Section 3 to deduce the general case. Indeed, for any separable subspace $Z$ of $X$, if we call $E$ the closed linear space spanned by $Z$ and $Y$, since $(E / Y)^{*}$ is separable we have

$$
S z(E) \leq S z(E / Y) . S z(Y) \leq S z(X / Y) . S z(Y)
$$

So

$$
S z(Z) \leq S z(X / Y) . S z(Y)
$$

Hence, by Proposition 3.1

$$
S z(X) \leq S z(X / Y) . S z(Y)
$$

Therefore, from now on, we will assume that $Y^{\perp}$ is separable and we will denote by $\mathcal{V}=\left(V_{n}\right)_{n=1}^{\infty}$ a basis of open sets for $\left(B_{Y^{\perp}}, \sigma\left(Y^{\perp}, X / Y\right)\right)$.

LEMMA 6.2. Let $\varepsilon>0, F=3 B_{Y^{\perp}}$ and $B=F+\frac{\varepsilon}{3} B_{X^{*}}$. For any ordinal $\alpha$ :

$$
B_{\varepsilon}^{[\omega \cdot \alpha]} \subseteq F_{\varepsilon / 3}^{[\alpha]}+\frac{\varepsilon}{3} B_{X^{*}}
$$


Proof. We will prove this by transfinite induction on $\alpha$.

By definition of $B$, it is true for $\alpha=0$.

Assume this property is true for any $\beta<\alpha$.

If $\alpha$ is a limit ordinal, we have that

$$
B_{\varepsilon}^{[\omega \cdot \alpha]}=\bigcap_{\beta<\alpha} B_{\varepsilon}^{[\omega \cdot \beta]} \subseteq \bigcap_{\beta<\alpha}\left(F_{\varepsilon / 3}^{[\beta]}+\frac{\varepsilon}{3} B_{X^{*}}\right)=F_{\varepsilon / 3}^{[\alpha]}+\frac{\varepsilon}{3} B_{X^{*}},
$$

because $\left(F_{\varepsilon / 3}^{[\beta]}\right)_{\beta<\alpha}$ is a decreasing family of $\sigma\left(Y^{\perp}, X / Y\right)$-compact sets.

If $\alpha=\beta+1$ : let $\left(V_{n_{i}(\alpha)}\right)_{i=1}^{\infty}=\left\{V \in \mathcal{V}\right.$ such that $V \cap F_{\varepsilon / 3}^{[\beta]} \neq \emptyset$ and $\left.\operatorname{diam}\left(V \cap F_{\varepsilon / 3}^{[\beta]}\right) \leq \frac{\varepsilon}{3}\right\}$. We will show by induction that for any $k \geq 1$ :

$$
B_{\varepsilon}^{[\omega \cdot \beta+k]} \subseteq\left(F_{\varepsilon / 3}^{[\beta]} \backslash \bigcup_{i=1}^{k} V_{n_{i}(\alpha)}\right)+\frac{\varepsilon}{3} B_{X^{*}}
$$

If we assume that this is true for $k$, we have that $B_{\varepsilon}^{[\omega \cdot \beta+k]} \backslash\left[\left(F_{\varepsilon / 3}^{[\beta]} \backslash \bigcup_{i=1}^{k+1} V_{n_{i}(\alpha)}\right)+\frac{\varepsilon}{3} B_{X^{*}}\right]$ is a $\sigma\left(X^{*}, X\right)$-open subset of $B_{\varepsilon}^{[\omega \cdot \beta+k]}$ and is included in $\left(V_{n_{k+1}(\alpha)} \cap F_{\varepsilon / 3}^{[\beta]}\right)+\frac{\varepsilon}{3} B_{X^{*}}$. So its diameter is $\leq \varepsilon$. Therefore $B_{\varepsilon}^{[\omega \cdot \beta+k+1]} \subseteq\left(F_{\varepsilon / 3}^{[\beta]} \backslash \bigcup_{i=1}^{k+1} V_{n_{i}(\alpha)}\right)+\frac{\varepsilon}{3} B_{X^{*}}$.

It follows from these inclusions that

$$
B_{\varepsilon}^{[\omega \cdot \alpha]} \subseteq\left(F_{\varepsilon / 3}^{[\beta]} \backslash \bigcup_{i=1}^{\infty} V_{n_{i}(\alpha)}\right)+\frac{\varepsilon}{3} B_{X^{*}}=F_{\varepsilon / 3}^{[\alpha]}+\frac{\varepsilon}{3} B_{X^{*}}
$$

Let $q$ be the quotient map from $X^{*}$ to $X^{*} / Y^{\perp}$. We have the following lemma.

LEMMA 6.3. For any ordinal $\alpha, q\left(\left(B_{X^{*}}\right)_{\varepsilon}^{\left[\gamma_{\varepsilon} \cdot \alpha\right]}\right) \subseteq\left(B_{X^{*} / Y^{\perp}}\right)_{\varepsilon / 4}^{[\alpha]}$,

where $\gamma_{\varepsilon}=\omega \cdot S_{\varepsilon / 3}(F)=\omega \cdot S z\left(X / Y, \frac{\varepsilon}{9}\right)$.

Proof. Again the proof is a transfinite induction.

Since $q\left(B_{X^{*}}\right)=B_{X^{*} / Y^{\perp}}$, the case $\alpha=0$ is clear.

Assume this is true for any ordinal $\alpha<\beta$.

If $\alpha$ is a limit ordinal, it is easy to check that the property considered is therefore true for $\alpha$.

If $\alpha=\beta+1$ : let $x^{*} \in B_{X^{*}}$ so that $q x^{*} \notin\left(B_{X^{*} / Y^{\perp}}\right)_{\varepsilon / 4}^{[\alpha]}$. We need to prove that $x^{*} \notin\left(B_{X^{*}}\right)_{\varepsilon}^{\left[\gamma_{\varepsilon} \cdot \alpha\right]}$, so we may assume that $x^{*} \in\left(B_{X^{*}}\right)_{\varepsilon}^{\left[\gamma_{\varepsilon} \cdot \beta\right]}$ and then, by induction hypothesis, 
$q x^{*} \in\left(B_{X^{*} / Y^{\perp}}\right)_{\varepsilon / 4}^{[\beta]}$. Therefore there is a $\sigma\left(X^{*} / Y^{\perp}, Y\right)$-neighborhood $\tilde{V}$ of $q x^{*}$ such that $\operatorname{diam}\left(\tilde{V} \cap\left(B_{X^{*} / Y^{\perp}}\right)_{\varepsilon / 4}^{[\beta]}\right) \leq \frac{\varepsilon}{4}$. $\tilde{V}$ defines a $\sigma\left(X^{*}, X\right)$-neighborhood $V$ of $x^{*}$, and

$$
V \cap\left(B_{X^{*}}\right)_{\varepsilon}^{\left[\gamma_{\varepsilon} \cdot \beta\right]} \subseteq x^{*}+\left(3 B_{Y^{\perp}}+\frac{\varepsilon}{3} B_{X^{*}}\right) .
$$

By Lemma 6.2, $\left(3 B_{Y^{\perp}}+\frac{\varepsilon}{3} B_{X^{*}}\right)_{\varepsilon}^{\left[\gamma_{\varepsilon}\right]}=\emptyset$.

Therefore $x^{*} \notin\left(B_{X^{*}}\right)_{\varepsilon}^{\left[\gamma_{\varepsilon} \cdot \beta+\gamma_{\varepsilon}\right]}=\left(B_{X^{*}}\right)_{\varepsilon}^{\left[\gamma_{\varepsilon} \cdot \alpha\right]}$.

Proof of Theorem 6.1. We deduce directly from Lemma 6.3 that, for any $\varepsilon>0$ :

$$
S z(X, \varepsilon) \leq \omega \cdot S z\left(X / Y, \frac{\varepsilon}{9}\right) . S z\left(Y, \frac{\varepsilon}{4}\right)
$$

We will use the following easy and technical fact :

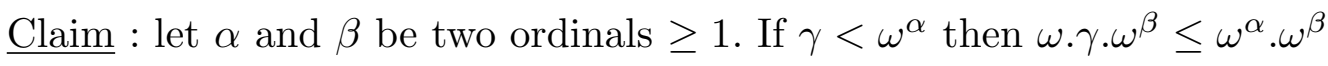

We want now to prove that $S z(X) \leq S z(X / Y) \cdot S z(Y)$. It is clear that if $\operatorname{dim}(Y)$ is finite then $S z(X)=S z(X / Y)$ and that if $\operatorname{dim}(X / Y)$ is finite then $S z(X)=S z(Y)$. Therefore we may assume that $S z(Y) \geq \omega$ and that $S z(X / Y) \geq \omega$. Then, if we combine the claim above with Proposition 5.4 we can conclude that $S z(X) \leq S z(X / Y) . S z(Y)$.

We will end this section with a slight improvement of the above inequality in the case where $Y$ is complemented in $X$. This will allow us to compute $S z(X)$ in some particular cases.

LEMMA 6.4. Let $X$ a Banach space and $Y$ a complemented subspace of $X$. If $S z(Y)<\omega_{1}$ and $S z\left(\frac{X}{Y}\right)<\omega_{1}$, then there exists a constant $C>0$ such that:

$$
\text { for any } \varepsilon>0, S z(X, \varepsilon) \leq S z\left(Y, \frac{\varepsilon}{C}\right) . S z\left(\frac{X}{Y}, \frac{\varepsilon}{C}\right) \text {. }
$$

Proof : It is enough to show that if $X=Y \oplus_{1} Z$, then for any $\varepsilon>0$ : $S z(X, \varepsilon) \leq S z(Y, \varepsilon) . S z(Z, \varepsilon)$. This can be done by a straightforward double transfinite induction.

Remark: Now it is not difficult to see that if $S z(Y) \leq \omega$ and $\operatorname{dim}(Z)=\infty$, then $S z(X)=S z(Z)$.

If we combine this remark with Proposition 3.1, we get the following result : 
PROPOSITION 6.5. Let $X$ be a Banach space and $Y$ be an infinite codimensional subspace of $X$ isomorphic to $c_{0}(\mathbb{N})$.

$$
\text { If } S z\left(\frac{X}{Y}\right)<\omega_{1} \text {, then } S z(X)=S z\left(\frac{X}{Y}\right) \text {. }
$$

Proof. By Proposition 3.1, it is enough to show that for any separable subspace $E$ of $X$ containing $Y$ and such that $Y$ is of infinite codimension in $E$, we have $S z(E) \leq S z\left(\frac{X}{Y}\right)$. But Sobczyk's theorem (see [So]) implies that $Y$ is complemented in $E$. Moreover it is easy to check that $S z\left(c_{0}(\mathbb{N})\right)=\omega$. Therefore, by the above remark, $S z(E)=S z\left(\frac{E}{Y}\right) \leq S z\left(\frac{X}{Y}\right)$.

Example : Let $J L$ be the space constructed by W.B. Johnson et J. Lindenstrauss (see [J-L] for the definition and the main properties of this space).

$J L$ contains a subspace $Y$ isometric to $c_{0}(\mathbb{N})$ and such that $\frac{J L}{Y}$ is isometric to $l_{2}(\Gamma)$, where $\Gamma$ is a certain uncountable set.

Thus, by Proposition 6.5, $S z(J L)=S z\left(l_{2}(\Gamma)\right)$.

But $l_{2}(\Gamma)$ is uniformly convex, so $S z\left(l_{2}(\Gamma)\right)=\omega=S z(J L)$.

\section{Acknowledgements.}

I would like to thank G. Godefroy for many helpful suggestions and B. Bossard for sending me a manuscript of his work. I also wish to thank the University of MissouriColumbia, where part of this work was completed. 


\section{REFERENCES}

[B] Bossard B., in preparation.

[Del ] Dellacherie C., Les dérivations en théorie descriptive des ensembles et le théorème de la borne. Séminaire de Probabilités XI, Université de Strasbourg, Lecture Notes in Math., Springer, vol 581 (1977), 34-46.

[Dev ] Deville R., Problèmes de renormage, J. of Funct. Anal. 68 (1986), 117-129.

[E-W] Edgar C.A. And Wheeler R.F., Topological properties of Banach spaces. Pac. J. of Math. ,115, 2 (1984).

[ J ] James R.C., A separable somewhat reflexive Banach space with non separable dual, Bull. A.M.S. , 80 (1974), 738-743.

[J-L ] Johnson W.B. And Lindenstrauss J., Some Remarks on weakly compactly generated Banach spaces, Israel J. Math. 17, 219-230.

[J-R] Johnson W.B. And Rosenthal H.P., On $w^{*}$-basic sequences and their applications to the study of Banach spaces, Studia Math. 43 (1972), 77-92.

[L ] Lancien G., Dentability indices and locally uniformly convex renormings, to appear in Rocky Mountain J. of Math.

[L-S ] Lindenstrauss.J. and Stegall C., Examples of separable spaces which do not contain $\ell_{1}$ and whose duals are non separable, Studia Math. , 54 (1975), $81-105$.

[Sa ] Samuel C., Indice de Szlenk des $\mathcal{C}(K)$ Publications mathématiques de l'Université Paris VI , Séminaire de Géométrie des espaces de Banach, (1983), tome 1, 81-91.

[Se] Sersouri A., Lavrientiev index for Banach spaces C.R. Acad. Sci. Paris Sér. I Math., 309 (1989), 2, 95-99.

[So] Sobczyk A., Projection of the space $m$ on its subspace $c_{0}$, Bull. A.M.S. , 47 (1941), 938-947.

[Sz ] SzLenk W., The non existence of a separable reflexive Banach space universal for all separable reflexive Banach spaces, Studia Math., 30 (1968), 53-61. 